\title{
Oxaliplatin-Induced Leukocytoclastic Vasculitis under Adjuvant Chemotherapy for Colorectal Cancer: Two Cases of a Rare Adverse Event
}

\author{
Henriette Quack $^{a} \quad$ Luise Erpenbeck, $^{b, f}$ Hendrik A. Wolff ${ }^{c}$ \\ Thilo Sprenger $^{\mathrm{a}} \quad$ Cornelia S. Seitz $^{\mathrm{b}}$ Michael P. Schö ${ }^{\mathrm{b}}$ \\ Steffen Neumann $^{\mathrm{e}}$ Kathrin Stanek $^{\mathrm{a}} \quad$ B. Michael Ghadimi ${ }^{\mathrm{a}}$ \\ Beate Michels $^{\mathrm{a}}$ Peter Middel $^{\mathrm{d}}$ Inga-Marie Schaefer ${ }^{\mathrm{d}}$ Torsten Liersch $^{\mathrm{a}}$ \\ Lena-Christin Conradi ${ }^{a}$ \\ Departments of ${ }^{\mathrm{a}}$ General and Visceral Surgery, ${ }^{\mathrm{b}}$ Dermatology, Venereology and \\ Allergology and ' Radiooncology, and ${ }^{\mathrm{d}}$ Institute for Pathology, University Medical Center, \\ Göttingen, and ${ }^{\mathrm{e} D}$ Department of Haematology and Oncology, Klinikum Wolfsburg, \\ Wolfsburg, Germany; ${ }^{f}$ Immune Disease Institute, Harvard Medical School/Children's \\ Hospital, Boston, Mass., USA
}

\section{Key Words}

Leukocytoclastic vasculitis · Oxaliplatin · Colorectal cancer · Chemotherapy-associated toxicity - Glomerulonephritis

\begin{abstract}
Leukocytoclastic vasculitis is a multicausal systemic inflammatory disease of the small vessels, histologically characterized by inflammation and deposition of both nuclear debris and fibrin in dermal postcapillary venules. The clinical picture typically involves palpable purpura of the lower legs and may be associated with general symptoms such as fatigue, arthralgia and fever. Involvement of the internal organs, most notably the kidneys, the central nervous system or the eyes, is possible and determines the prognosis. Oxaliplatin-induced leukocytoclastic vasculitis is a very rare event that limits treatment options in affected patients. We report 2 patients who developed the condition under chemotherapy for advanced rectal and metastatic colon carcinoma, respectively; a termination of the therapy was therefore necessary. While current therapies for colorectal cancer include the combination of multimodal treatment with new and targeted agents, rare and unusual side effects elicited by established agents also need to be taken into account for the clinical management.
\end{abstract}

Lena-Christin Conradi, MD

Department of General Surgery, University Medical Center Göttingen

Robert-Koch-Strasse 40

DE-37075 Göttingen (Germany)

E-Mail lena.conradi@med.uni-goettingen.de 
Quack et al.: Oxaliplatin-Induced Leukocytoclastic Vasculitis under Adjuvant

Chemotherapy for Colorectal Cancer: Two Cases of a Rare Adverse Event

(C) 2013 S. Karger AG, Basel

\section{Background}

The current standard of treatment for locally advanced rectal cancer (UICC stages II and III) includes a 5-fluorouracil (5-FU)-based preoperative radiochemotherapy (RCT), total mesorectal excision, and postoperative chemotherapy [1,2]. Several randomized clinical trials during the last decades have demonstrated that neoadjuvant 5-FU-based RCT significantly improves locoregional control of rectal cancer [3, 4]. Today, the limitation of the prognosis of individual patients is mainly determined by the occurrence of distant metastases in $3040 \%$ of patients. This led to the intensification of systemic therapy through integration of platinum derivatives [5], an approach that not only promises a therapeutic benefit but simultaneously bears the risk of increased toxicity [6]. Subsequently, to avoid unnecessary toxicity, more individualized and risk-adapted treatment approaches are needed and are currently being validated in several ongoing clinical trials. However, in rectal cancer, reliable biomarkers to predict the response to neoadjuvant RCT, individual prognosis and occurrence of treatment-associated toxicity have not yet been established as part of the clinical routine [7].

The adjuvant treatment strategy with 5-FU in combination with leucovorin and oxaliplatin is well established and has been used for nearly 10 years in the adjuvant treatment of UICC III colon cancer [8]. The neoadjuvant and adjuvant treatment with FOLFOX against locally advanced rectal cancer (UICC II and III) is integrated in several randomized phase III multicenter trials such as the CAO/ARO/AIO-04 trial of the German Rectal Cancer Study Group [7].

The CAO/ARO/AIO-04 regimen consists of intravenous oxaliplatin $\left(80 \mathrm{mg} / \mathrm{m}^{2}\right)$ combined with leucovorin $\left(400 \mathrm{mg} / \mathrm{m}^{2}\right)$ and subsequent 5 -FU infusion $\left(2,400 \mathrm{mg} / \mathrm{m}^{2}\right)$, repeated every 14 days over 4 months ( 8 cycles). Oxaliplatin is a platinum derivative whose therapeutic effect is based on alkylating effects. The platinum compound binds to DNA forming cross-links, which inhibit DNA replication and transcription, and so result in cell death $[9,10]$.

Oxaliplatin is mainly eliminated renally. For patients with chronic renal failure (creatinine clearance $<30 \mathrm{ml} / \mathrm{min}$ ), a dose reduction is necessary (starting dose of $65 \mathrm{mg} / \mathrm{m}^{2}$ ). Common adverse reactions of oxaliplatin (over 10\%) include central neurological, gastrointestinal, hematologic, and peripheral neuropathic reactions [11]. Typical side effects of oxaliplatin are a grade 3-4 neutropenia and in rare cases neutropenic fever. The myelosuppressing side effect can induce mild anemia and thrombocytopenia.

The main gastrointestinal adverse effects are nausea, vomiting, and diarrhea [12]. The peripheral neuropathy is very common; it might affect the patient's quality of life and might therefore lead to dose reductions [13]. The acute form is characterized by peripheral, acral and facial paresthesia and pain, commonly being exacerbated by exposure to the cold. The symptoms usually subside spontaneously after hours or days. The chronic side effects of neuropathy grade 34 with decreased distal sensation and proprioception affect the extremities and are reversible in most patients [14]. Of note, cutaneous symptoms after application of oxaliplatin are very rare [13].

Here we report on 2 cases of oxaliplatin-induced leukocytoclastic vasculitis with kidney involvement, a side effect which - to the best of our knowledge - was only reported in a single case before [15]. Written informed consent was obtained from the patients for the publication of this paper and any accompanying images. 
Quack et al.: Oxaliplatin-Induced Leukocytoclastic Vasculitis under Adjuvant

Chemotherapy for Colorectal Cancer: Two Cases of a Rare Adverse Event

\section{Case 1}

A 75-year-old man presented with a locally advanced adenocarcinoma of the rectum (37 $\mathrm{cm}$ above the anal verge, endoscopically staged as uT3 uN). As he had received interstitial brachytherapy (>145 Gy) 2 years before as a treatment for prostate cancer (cT2b, cNx, cM0, Gleason score 6 ), the standard treatment of neoadjuvant RCT with 50.4 Gy and concomitant 5-FU (+/- oxaliplatin) was not possible. An abdomino-perineal rectum exstirpation with implantation of a descendostoma was performed to remove the rectal cancer. The histopathological work-up revealed a pT3a, pN2b (21/31), cM0, L1, V0, G3, R0 (UICC IIIB) adenocarcinoma and thus adjuvant chemotherapy was strongly suggested according to the vote of the institutional interdisciplinary tumor board.

Eight weeks after surgery, the first cycle of adjuvant chemotherapy with 5-FU, leucovorin and oxaliplatin (FOLFOX-6) was scheduled. The cycle consisted of a bimonthly regimen with intravenous oxaliplatin $\left(80 \mathrm{mg} / \mathrm{m}^{2}\right)$ and leucovorin $\left(400 \mathrm{mg} / \mathrm{m}^{2}\right)$ administered on day 1. 5 -FU $2,400 \mathrm{mg} / \mathrm{m}^{2}$ followed on day 1 as a continuous infusion for $46 \mathrm{~h}$. During the following 3 days, the patient developed fever that resolved spontaneously. Fourteen days later, the patient showed disseminated petechiae with secondary hemorrhage, along with edema on both legs and interdigital ulceration. He reported that he had experienced arthralgia in his knees and elbows 4 days after the first dose of chemotherapy. His blood count showed microcytic, hypochromic anemia, leukocytosis of $13,900 / \mu \mathrm{l}$, elevated Creactive protein of $90.1 \mathrm{mg} / \mathrm{l}$, and an increase in the retention parameters (urea $25 \mathrm{mg} / \mathrm{dl}$ ), indicating an affliction of the kidneys. The urine analysis was normal apart from an alkaline $\mathrm{pH}$ of 7.5.

The chemotherapy was interrupted and the patient was admitted to the Department of Dermatology. On examination, there was palpable purpura with petechial hemorrhage on the lower limb in addition to massive edema on both lower legs (fig. 1). Some petechiae were also found on the lower arms and the abdomen. We suspected drug toxicity to be the cause of the skin lesions. A skin biopsy confirmed the diagnosis of leukocytoclastic vasculitis. The patient did not complain about abdominal pain and 3 tests for occult blood in the stool were negative. There was no evidence for a systemic vasculitis reaction. After 2 days, the renal parameters were rising (creatinine $1.33 \mathrm{mg} / \mathrm{dl}$; normal range $0.5-1.0 \mathrm{mg} / \mathrm{dl}$ ) and the patient developed proteinuria of $1,200 \mathrm{mg} /$ day in the 24-hour urine collection. Because of the putative renal involvement, therapy with a high-dose corticosteroid (prednisolone 80 $\mathrm{mg}$ /day), a proton pump inhibitor (pantoprazole $40 \mathrm{mg} /$ day) and osteoporosis prophylaxis (calcium carbonate and cholecalciferol $500 \mathrm{mg}$ and $10 \mu \mathrm{g}$ b.i.d., respectively) was initiated. In addition, the patient was prescribed an ACE-inhibitor (ramipril $2.5 \mathrm{mg} /$ day) for renal protection.

An infectious origin of the leukocytoclastic vasculitis was considered implausible because there were no respective clinical symptoms and serological tests were negative for hepatitis A, B and C as well as for an infection with streptococci. The rheumatoid factors, C3c $(1.37 \mathrm{~g} / \mathrm{l}), \mathrm{C} 4(0.25 \mathrm{~g} / \mathrm{l}), \mathrm{p}-\mathrm{ANCA}-\mathrm{IF}(<1: 20)$, and ANA-IF $(>1: 80)$, were not elevated and no circulating immune complexes were detected.

A punch biopsy from the active area of the frontal aspect of the right lower leg showed typical features of leukocytoclastic vasculitis (fig. 2). There were lymphocytic infiltrates with numerous neutrophils as well as 'nuclear dust' in the higher corium, extravasated erythrocytes, focal small-vessel edema, endothelial cell swelling within the vessels and fibrinoid deposition. Direct immunofluorescence revealed $\operatorname{IgM}, \operatorname{IgA}, \mathrm{C} 3$, and fibrinogen deposition in the small vessels of the dermis. 
In our opinion, there was strong evidence for drug-induced toxicity as the cause of the vasculitis, so chemotherapy with oxaliplatin was discontinued. The patient was treated with prednisolone at a decreasing dosage for the next 6 weeks. Under the steroid therapy, the vasculitis lesions disappeared rapidly.

The patient's general practitioner continuously monitored creatinine clearance and proteinuria. After 2 weeks, there was evidence of hematuria ( $>20$ erythrocytes) and proteinuria $(745 \mathrm{mg} / \mathrm{l})$. The urine analysis showed an increase in renal parameters with creatinine $2.41 \mathrm{mg} / \mathrm{dl}$, urea $43 \mathrm{mg} / \mathrm{dl}$, eGFR 26.3 , a proteinuria of $1,800 \mathrm{mg} /$ day in a 24 -hour urine collection, and a creatinine clearance of $40.1 \mathrm{ml} / \mathrm{min}$. The patient was admitted to the Department of Nephrology with the clinical diagnosis of acute renal failure, presumably as a consequence of acute glomerulonephritis. The percutaneous renal biopsy showed a focal segmental extravascular necrotizing proliferating glomerulonephritis. Because GBMantibodies $(0.4 \mathrm{U} / \mathrm{ml})$ or cryoglobulins $(<0.2 \%)$ were not detectable, the most likely cause of renal failure was a renal manifestation of the leukocytoclastic vasculitis. Under high-dose steroid therapy (prednisolone $250 \mathrm{mg}$ for 3 days followed by tapered doses), the renal function improved slowly.

Unfortunately, during further follow-up, the patient had developed tumor progression with pulmonary metastases at the 12 -month follow-up visit. Consequently, a palliative regimen with FOLFIRI (5-FU, leucovorin and irinotecan) was scheduled. Under this treatment, no side effects were apparent and the metastases remained stable for 8 months. Recent MRI and CT scans revealed a progressive disease with new metastasis-like lesions in the thoracic spine and suspect lymph nodes in the retroperitoneal, iliacal and inguinal region.

\section{Case 2}

We also treated a 71-year-old male patient with similar symptoms after chemotherapy with FOLFOX-6 and bevacizumab for colon cancer with hepatic metastases [pT3, pN0 (0/33), pM1 (hepatic), G2, R0]. Initially, he had received surgical treatment with a right hemicolectomy and an atypical liver resection of some of the lesions. After the subsequent postoperative chemotherapy with FOLFOX- 6 and bevacizumab for 4 cycles, restaging was performed. Due to the remaining hepatic metastases, a right hemihepatectomy was performed. Postoperatively, the patient developed ubiquitous petechial hemorrhage. Except for increased S-ANA-IF activity (1:320), the rheumatoid factors were negative. A punch biopsy from an active hemorrhagic lesion again confirmed the diagnosis of a leukocytoclastic vasculitis. Shortly after diagnosis, the patient showed clinical symptoms of acute renal failure and his renal parameters increased to a serum creatinine of $2.5 \mathrm{mg} / \mathrm{dl}$ and an urea of $69 \mathrm{mg} / \mathrm{dl}$, necessitating forced diuresis in addition to systemic corticosteroids for the next 2 months. The nephrotic syndrome with microhematuria persisted for 2 months of continued cortisone therapy.

A renal biopsy was repeated 2 months later and showed an IgA nephritis with focal segmental and global glomerulosclerosis. As treatment for the chronic renal failure grade III, a continuous cortisone therapy was prescribed. Assuming that drug toxicity was the cause of the vasculitis, the supporting chemotherapy was prematurely terminated.

Within the following 12 months, the patient regretfully developed new metastases in the right lung segment and also showed bifocal liver metastases in the remaining left liver lobe. Therefore, he was treated with a modified FOLFIRI 14. The dose reduction was $75 \%$ and he received additional fluids for renal protection. Under this treatment, the patient again 
suffered from acute or chronic renal failure, which made hemodialysis necessary. The cortisone therapy was continued.

\section{Conclusions}

Oxaliplatin is a third-generation platinum alkylating agent currently used to treat a great number of malignancies, including both locally advanced and metastatic colorectal cancer. Unfortunately, the use of oxaliplatin is associated with a plethora of adverse effects. While some of these effects, like nausea or thrombocytopenia, are common reactions to many cytostatic drugs, oxaliplatin has been reported to induce some rather unique side effects. With the report of these 2 cases, we have documented a very rare oxaliplatin-induced side effect with a potentially dramatic impact on the affected patients. Physicians should be aware of this rare, yet potentially detrimental adverse effect of oxaliplatin when employing it as a standard chemotherapeutic substance or as a part of novel combination therapies.

To our knowledge, there has been only 1 case report prior to our observations reporting leukocytoclastic vasculitis as an adverse effect of treatment with oxaliplatin [15].

\section{Acknowledgements}

The authors thank Birgit Jünemann for her excellent technical assistance and Lesley Cowan for the proofreading of this paper.

\section{Disclosure Statement}

The authors declare no conflict of interest.

\section{References}

1 Rödel C, Sauer R: Neoadjuvant radiotherapy and radiochemotherapy for rectal cancer. Recent Results Cancer Res 2005;165:221-230.

2 Heald RJ: Total mesorectal excision (TME). Acta Chir Iugosl 2000;47(4 suppl 1):17-18.

-3 Sauer R, Becker H, Hohenberger W, et al: Preoperative versus postoperative chemoradiotherapy for rectal cancer. N Engl J Med 2004;351:1731-1740.

-4 Sauer R, Liersch T, Merkel S, et al: Preoperative versus postoperative chemoradiotherapy for locally advanced rectal cancer: results of the German CAO/ARO/AIO-94 randomized phase III trial after a median follow-up of 11 years. J Clin Oncol 2012;30:1926-1933.

5 Rödel C, Hofheinz R, Liersch T: Rectal cancer: state of the art in 2012. Curr Opin Oncol 2012;24:441-447.

-6 Wolff HA, Conradi LC, Schirmer M, et al: Gender-specific acute organ toxicity during intensified preoperative radiochemotherapy for rectal cancer. Oncologist 2011;16:621-631.

7 Liersch T, Rothe H, Ghadimi BM, et al: Individualizing treatment for locally advanced rectal cancer (in German). Chirurg 2009;80:281-293.

8 Martin MJ: Current stage-specific chemotherapeutic options in colon cancer. Expert Rev Anticancer Ther 2005;5:695-704.

-9 Alcindor T, Beauger N: Oxaliplatin: a review in the era of molecularly targeted therapy. Curr Oncol 2011;18:18-25.

10 Rödel C, Liersch T, Becker H, et al: Preoperative chemoradiotherapy and postoperative chemotherapy with fluorouracil and oxaliplatin versus fluorouracil alone in locally advanced rectal cancer: initial results of the German CAO/ARO/AIO-04 randomised phase 3 trial. Lancet Oncol 2012;13:679-687. 


\section{Case Reports in Oncology}

\begin{tabular}{l|l}
\hline Case Rep Oncol 2013;6:609-615 \\
\hline DOI: $10.1159 / 000357166$ & $\begin{array}{l}\text { C 2013 S. Karger AG, Basel } \\
\text { www.karger.com/cro }\end{array}$ \\
\hline
\end{tabular}

Quack et al.: Oxaliplatin-Induced Leukocytoclastic Vasculitis under Adjuvant Chemotherapy for Colorectal Cancer: Two Cases of a Rare Adverse Event

11 Takimoto CH, Remick SC, Sharma S, et al: Dose-escalating and pharmacological study of oxaliplatin in adult cancer patients with impaired renal function: a National Cancer Institute Organ Dysfunction Working Group Study. J Clin Oncol 2003;21:2664-2672.

-12 Culy CR, Clemett D, Wiseman LR: Oxaliplatin. A review of its pharmacological properties and clinical efficacy in metastatic colorectal cancer and its potential in other malignancies. Drugs 2000;60:895-924.

13 Hartmann JT, Lipp HP: Toxicity of platinum compounds. Expert Opin Pharmacother 2003;4:889-901.

14 Weickhardt A, Wells K, Messersmith W: Oxaliplatin-induced neuropathy in colorectal cancer. J Oncol 2011, DOI: $10.1155 / 2011 / 201593$.

15 Hill SE, Phillips R, Francis N, et al: Small-vessel vasculitis following treatment with combination 5fluorouracil/folinic acid and oxaliplatin. Clin Exp Dermatol 2009;34:103-105.
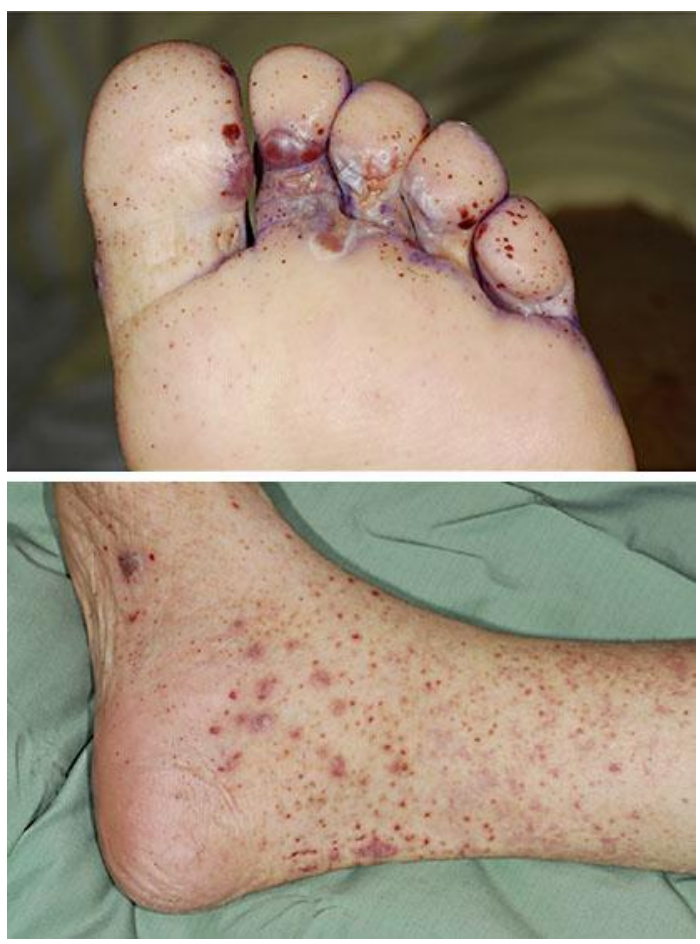

Fig. 1. Macroscopic lesions in leukocytoclastic vasculitis. Skin lesions of patient 2 after exposure to oxaliplatin in a FOLFOX regimen against metastatic colon cancer. 
Quack et al.: Oxaliplatin-Induced Leukocytoclastic Vasculitis under Adjuvant Chemotherapy for Colorectal Cancer: Two Cases of a Rare Adverse Event

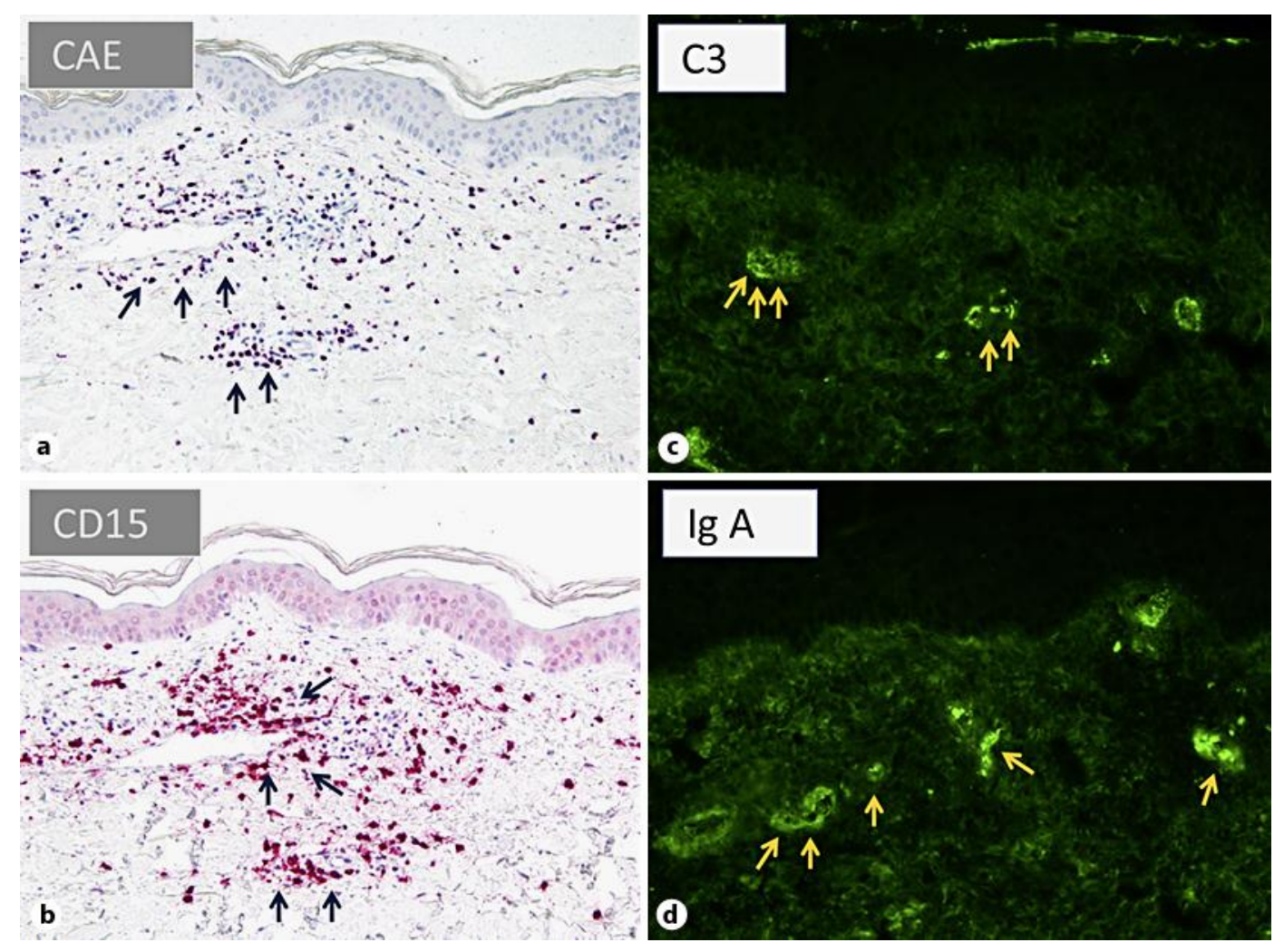

Fig. 2. Immunohistochemical staining for chloroacetate esterase, CD15 and direct immunfluorescence for C3 and IgA. Skin biopsy from an area with macroscopic lesions. Chloroacetate esterase staining (CAE; a) and immunohistochemical staining for CD15 (b) showed accumulations of granulocytes surrounding the small vessel walls. Direct immunofluorescence showed complement factor C3 (c) and IgA deposits (d) within the small vessels of the papillary dermis. 